\section{Large Left Ventricular Thrombus in a Neonate - A Rare Complication of Severe Persistent Pulmonary Hypertension of the Neonate}

A girl weighing $3.6 \mathrm{~kg}$ was born at term via spontaneous delivery through thick meconium after an uneventful pregnancy to a healthy primigravida mother. Apgar scores were 4 and 7 at 1 and 5 minutes, respectively, and cord blood $\mathrm{pH}$ was 7.27 with base excess of -5 . She presented with respiratory distress and was diagnosed with meconium aspiration syndrome requiring chest drain, and mechanical ventilation. At 18 hours, she was diagnosed on echocardiography with persistent pulmonary hypertension of the neonate (PPHN), and inhaled nitric oxide (iNO) was added. She improved initially, did not require inotropes, and was off iNO, but she deteriorated suddenly on day 8 with pallor, hypertension and tachycardia - there were no concurrent central line or sepsis. Urgent echocardiography revealed severely reduced biventricular function and a large, $11 \times 10 \mathrm{~mm}$ left ventricular thrombus, which resolved with enoxaparin and milrinone. Cardiac function remains normal at the age of 2.5 years, without any personal or familial underlying cardiomyopathy or prothrombotic condition.

Treatment of PPHN consists of ventilation, iNO, and inotropic support. Thromboembolic events, of which $16 \%$ are intracardiac, occur with risk factors such as central access, extreme prematurity, and inflammation. Intracardiac thrombi in the

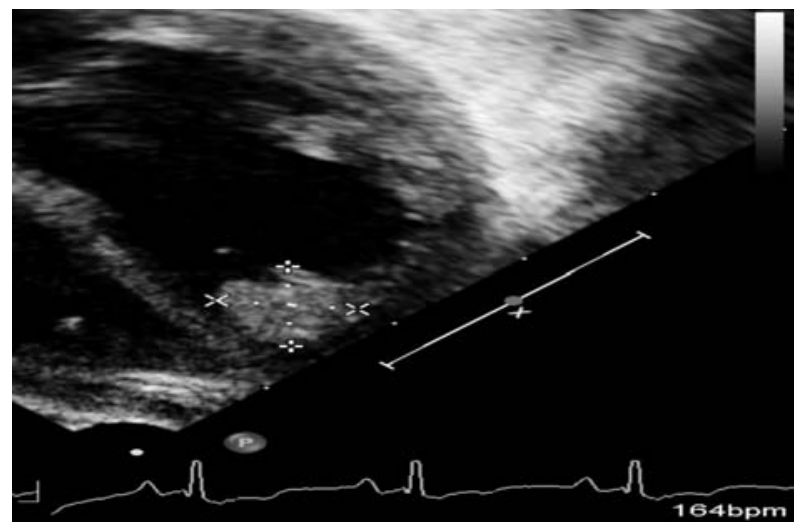

Fig. 1 Four chamber apical trans-thoracic echocardiographic image with left ventricular thrombus (with measurements).

absence of congenital heart disease, cardiomyopathy, or post cardiac surgery are rare. Symptoms for central arterial thrombosis may be absent, or subtle such as lethargy, thrombocytopenia, or mimic coarctation. Therapeutic recommendations depend on thrombus location, etiology, and must be weighed against bleeding risk.

\author{
Amelie STritzke, ${ }^{1,2 *}$ Lindsay Mills ${ }^{3}$ \\ From ${ }^{1}$ Section of Neonatology and ${ }^{3}$ Pediatric Cardiology, \\ Department of Pediatrics, University of Calgary; \\ ${ }^{2}$ Libin Cardiovascular Institute of Alberta; \\ Calgary, Alberta, Canada. \\ *Amelie.Stritzke@albertahealthservices.ca
}

\section{Language Barrier in Anganwadis in Eranakulam, Kerala}

The utilization of Integrated Child Development Services in Kerala was found to be less than optimum in our previous study in Njarackal, Eranakulam, Kerala [1]. We conducted a qualitative study in the same area between March and May, 2018, to determine the reasons for underutilization of services. The method consisted of a focus group discussion with 11 mothers with young children, and in-depth interviews with two anganwadi workers and a Child development program officer. The recorded interviews were transcribed, themes were identified and triangulated.

Most mothers and functionaries desired better physical infrastructure at the anganwadis, where the children could run around and play. The mothers felt that most anganwadis had very limited infrastructure, so children had to sit and sleep in a small room throughout the day. Most mothers were satisfied with the cleanliness, food and attention given by the staff at anganwadis. Despite many shortcomings, most mothers were willing to send their children to the anganwadi. Some mothers commented "children eat more when they are at anganwadi than at home."

The main reason for the parents not sending children beyond 4 years to the anganwadi was absence of English education. The medium of instruction in anganwadis is Malayalam, the local language and mother tongue of most children. English is not taught as part of nonformal education, except for the alphabet. Most parents conveyed their desire to admit their children into English medium schools in class I, where a major eligibility criterion is basic knowledge of English. Those students who attend pre-school at English-medium kindergartens have significant advantage over those who attend Malayalam-medium anganwadis. Hence, parents opined that they withdraw their children from the anganwadis and send them to private English medium kindergartens. Some mothers 